\title{
Research on Fault Diagnosis of Rotating Machinery of Vehicle Transmission
}

\author{
Tao Zhang, Huiyuan Xue a \\ Beijing Engineering Research Center for loT Software and Systems, Faculty of Information \\ Technology, Beijing University of Technology, Beijing, China \\ aCorresponding author: 1553810420@qq.com
}

\begin{abstract}
Keywords: Special Armored Vehicle Gearbox, Fault Diagnosis, EMD, Endpoint Effect, Feature Extraction, SVM.
\end{abstract}

\begin{abstract}
In this paper, the gearbox as the research object, under the conditions of strong interference signal acquisition and analysis, denoising, eigenvalue extraction, pattern recognition and fault prediction has very important practical significance. Aiming at the problem of end effect in Empirical Mode Decomposition (EMD), an improved method for the continuation of the even-extended cosine window function (EECW) of signal sequences is proposed. Firstly, the signal sequence is continually extended to realize the smooth transition between the extension data and the original signal, avoiding the jump of the instantaneous frequency of the signal. Secondly, there is the problem of continuation error for this extension method. (Or slow speed) to the internal development of data to ensure the correct decomposition of the signal valid data to improve the accuracy of the decomposition of the signal to achieve the improvement of EMD algorithm. Through the simulation analysis and fault diagnosis, it is shown that the method can effectively suppress EMD endpoint effect and realize the effective diagnosis of rotating machinery fault. The experimental results show that the proposed method has high precision and good performance
\end{abstract}

\section{Introduction}

A special vehicle transmission system application environment, as the core components of the transmission system gearbox, long-term to withstand large loads, strong shock, prone to all kinds of gears, bearings and shaft failure for the maintenance of armored vehicles great inconvenience.

The traditional denoising method is based on the characteristics of the actual signal, the statistical characteristics of noise and spectrum distribution of the law, the use of filters for signal noise reduction. The most commonly used method is based on the noise energy is generally concentrated in the high-frequency, and the signal spectrum is distributed in a limited range of this feature, using Fourier transform will be noisy signal to the frequency domain, and then use low-pass filter Denoising. When the noise and the signal frequency band overlap, such as the signal contains white noise, lowpass filter denoising effect is poor. The traditional denoising method is based on the theory of Fourier transform, there is the local characteristics of the protection signal and the contradiction between noise suppression. Wavelet transform has a good time-frequency localization characteristics, can solve this contradiction. Of course, the wavelet denoising method has been widely used in signal denoising and has achieved a very good application effect [1], becoming one of the main methods of signal denoising.

Rotating machinery fault diagnosis, often encountered such as transient, amplitude modulation or FM and other non-stationary, non-linear signal, extract and analyze the characteristics of these signals is the key to mechanical diagnosis. EMD is a new time-frequency analysis method proposed by Huang [2], which can decompose the complex signal into finite Intrinsic Mode Function (IMF), which is suitable for dealing with non-linear and non-stationary signals. Since the advent of EMD method [3], it has attracted the attention of many scholars, has been used in structural analysis, equipment diagnosis and other fields [4].

The quality of EMD analysis depends largely on the quality of EMD decomposition. The EMD decomposition of the method using cubic spline interpolation to obtain the instantaneous average of 
the signal, making this method there is a special end effect [5]. In addition, the Hilbert transform also exists in the process of endpoint effects, seriously affect the entire signal analysis results. In order to solve this problem, researchers have proposed some improved methods, such as image extension, neural network extension, similarity extremum extension, signal sequence even extension, support vector regression and so on[6] [7]. However, there is a common problem in these extension methods, that is, after the extension [8], the endpoint of the signal is still uncertain, so that as the decomposition progresses, there is still a divergence phenomenon at both ends of the envelope, ", Leading to its endpoint effect problem still exists [9].

In this paper, we propose an EMD end-point processing method combined with even-extension and cosine-window functions for signal sequences [10]. This method preserves the advantage of the signal sequence even extension method, through the window function processing, so that the extension part of the gradual reduction until zero, so that the extension error decreases. The effectiveness of the proposed method is verified by simulation analysis and mis-alignment fault diagnosis.

This paper presents a method to diagnose the failure of gearbox of armored vehicles by combining wavelet, improved EMD, and support vector machine (SVM) theory.

\section{Wavelet Denoising Method}

\subsection{Wavelet Denoising Theory}

First define the wavelet function, set the function $\psi$ in the infinite interval $(-\infty,+\infty)$, There is an energy limit, $L_{2}(R)$ is a function $\psi$ of the signal space. This generates the following family of functions $\left\{\psi_{\text {ab }}\right\}$ :

$$
\Psi_{\text {ab }}(\mathrm{t})=|\mathrm{a}|^{1 / 2} \Psi\left(\frac{t-b}{a}\right) \quad-\infty<b<+\infty, a>0
$$

Among them, $\psi_{\mathrm{ab}}(\mathrm{t})$ Called wavelet basis function, Called wavelet basis function. $\mathrm{b}$ is the translation coefficient, $\mathrm{a}$ is the expansion coefficient. Given function $\left.\mathrm{f}_{\mathrm{f}} \mathrm{x}\right)$ belong to the signal space $L_{2}(R)$, the inner product of the basis function can be obtained from the following formula:

$$
W T(\mathrm{f}(\mathrm{t}) ; \mathrm{a}, \mathrm{b})=\left\langle\mathrm{f}, \psi_{\mathrm{ab}}\right\rangle=|a|^{-1 / 2} \int_{R} \psi\left(\frac{t-b}{a}\right) d t
$$

\subsection{Noise Processing}

The vibration signal collected by the sensor can be simplified as the $m$ following function:

$$
x(t)-m_{1}(t)=h_{1}(t) s(t)=f(t)+\sigma e(t)
$$

Among them, $s(t)$ is the original signal, It consists of two parts, $e(t)$ for noise interference, $f(t)$ as a useful signal. Usually, Fault information is generally reflected in the low frequency band, the main components of noise interference is high-frequency signals. Wavelet denoising method is effective decomposition of high and low frequency components, leaving a useful part of the signal to eliminate interference components, to achieve the effect of noise.

In this paper, the original vibration signal shown in Figure 1:

Firstly, the original vibration signal is decomposed by N-layer wavelet. According to the actual situation, the threshold value can be set, and different coefficients can be set for the signal components of different frequency sections. Then select the useful signal layer, reconstruct the signal. The reconstructed signal can remove the interference components effectively, leaving the faulty information rich. Specific treatment is as follows:

The use of matlab software toolbox. First, use the toolbox in the ddencmp function to obtain the optimal standard threshold of the original vibration signal, and then do wdencmp function to deal with. This method is easy to implement, simple operation, the effect is not bad. Under the condition of normal rotor, the signal is analyzed, and wavelet de-noising is carried out.Figure 2 is the signal after wavelet denoising 

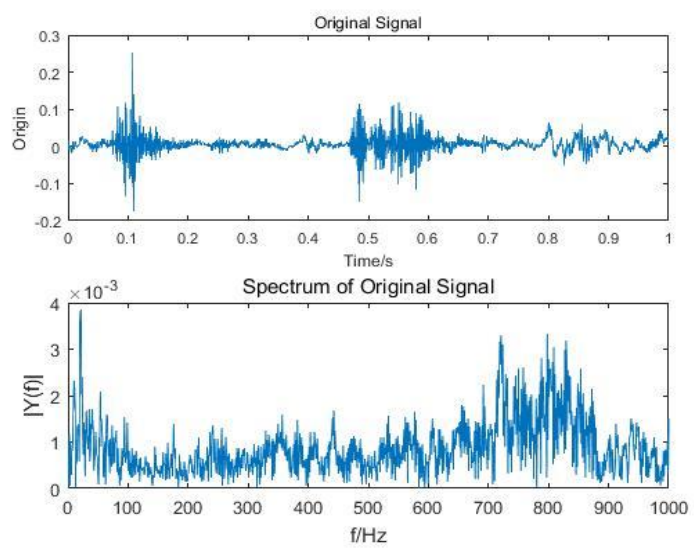

Fig.1 The original signal
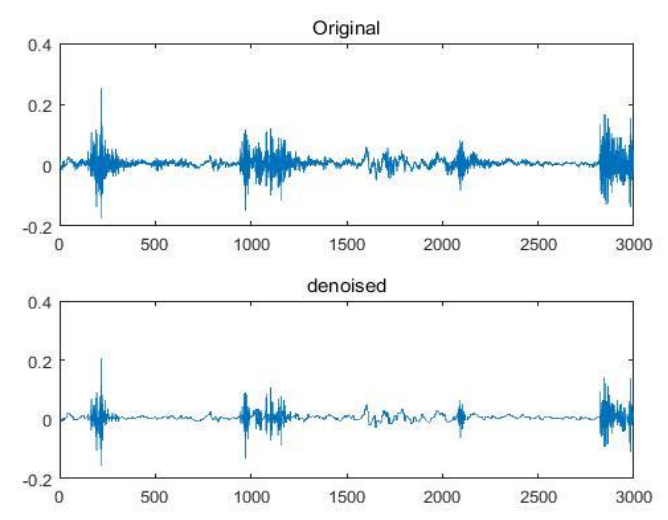

Fig. 2 Comparison of wavelet denoising

It can be seen that the wavelet denoising effect is good. After the original vibration signal is denoised by wavelet, the high-frequency interference component in the signal is reduced a lot, and the wavelet denoising method is used to preprocess the faulty vibration signal.

\section{Improved Emd Time - Frequency Analysis Method}

\subsection{EMD decomposition method}

EMD time-frequency analysis method is suitable for dealing with non-linear, non-stationary signal. The Hilbert-Huang transform consists of two processes: Empirical Mode Decomposition (EMD) and Hilbert Transform.

EMD method decomposition steps are:

(1)Determine all local extrema points of the signal.

(2)All the local maximum points are connected to form an upper envelope using a cubic spline; all local minimum points are connected to form a lower envelope. The upper and lower envelopes should envelope all data points.

(3) The average of the upper and lower envelopes is denoted by $m_{1}(t)$

(4) The difference between signals $x(t)$ and $m_{1}(t)$ is the first component, $h_{1}(t)$ is:

$x(t)-m_{1}(t)=h_{1}(t)$

If $h_{1}(t)$ is an IMF, then $h_{1}(t)$ is the first IMF component.

(5) $h_{1}(t)$ is not satisfied with the IMF conditions, the $h_{1}(t)$ as the original data, repeat the above steps, resulting in:

$h_{1}(t)-m_{11}(t)=h_{11}(t)$

Wherein, $m_{11}(t)$ is the average of the upper and lower envelopes of $h_{1}(t)$ After repeated screening $\mathrm{k}$ times, making $h_{1} k(t)$ for the IMF component, namely:

$$
H_{1}(k-1)(t)-m_{1} k(t)=h_{1} k(t)=c_{1}(t)
$$


The first IMF component $c_{1}(t)$ obtained from the original signal should contain the best range of the signal or the shortest periodic component. By separating $c_{1}(t)$ from $x(t)$, we have:

$r_{1}(t)=x(t)-c_{1}(t)$

Repeating the above steps with $r_{1}(t)$ as the original data yields the second IMF component $c_{2}(t)$ of $x(t)$. Repeat $\mathrm{n}$ times to get $\mathrm{n}$ IMFs of signal $x(t)$. This has:

$$
\left.\begin{array}{c}
r_{1}(t)-c_{2}(t)=r_{2}(t) \\
\ldots \\
r_{n-1}(t)-c_{n}(t)=r_{n}(t)
\end{array}\right\}
$$

When $r_{n}(t)$ becomes a monotone function can not be extracted from the IMF to meet the conditions of the component, the end of the cycle. This gives:

$$
x(t)=\sum_{j=1}^{n} c_{j}(t)+r_{n}(t)
$$

So the signal can be decomposed into $\mathrm{n}$ empirical mode, the residual function $r_{n}(t)$ represents the average trend of the signal. For each intrinsic mode function $c_{i}(t)$ do Hilbert transform:

$$
\begin{aligned}
\hat{c_{i}}(t) & =\frac{1}{\pi} \int_{-\infty}^{\infty} \frac{c_{i}(\tau)}{t-\tau} d \tau \\
z_{i}(t) & =c_{i}(t)+\hat{j c_{i}}(t)=a_{i}(t) e^{j \phi_{i}(t)}
\end{aligned}
$$

Then we get the amplitude function:

$$
a_{i}(t)=\sqrt{c_{i}(t)+{\hat{c_{i}^{2}}}^{2}(t)}
$$

And the phase function:

$$
\phi_{i}(t)=\arctan \frac{\hat{c}_{i}(t)}{c_{i}(t)}
$$

Further, the instantaneous frequency can be obtained:

$$
f_{i}(t)=\frac{1}{2 \pi} \omega_{i}(t)=\frac{1}{2 \pi} \times \frac{d \phi_{i}(t)}{d t}
$$

After the original signal after EMD decomposition, as shown in Figure 3

\subsection{EMD Decomposition of the Endpoint Effect}

EMD decomposition cannot guarantee the extreme point at the endpoint, resulting in the process of obtaining the average envelope, the spline interpolation will produce data fitting error. And with the decomposition of the error accumulation, from the endpoint to the gradual spread of serious data decomposition will make sense.

Figure 2 is the original signal without EMD decomposition, Figure 3 is the signal EMD decomposition results, the data from the gearbox rotor vibration at different frequencies by the vibration sensor to collect the real data.

Figure 4 shows the first IMF component obtained by EMD decomposition before the suppression of the boundary effect. In the figure, the solid line represents the IMF component and the broken line represents the first component $x_{1}(t)$ of the signal. It can be seen that both ends of the data have already appeared Serious distortion, the IMF component and there is a large error, if the error cannot be effectively controlled, the results become meaningless. Since the cubic spline interpolation requires the use of two neighboring points, the only way to solve the problem is to increase the appropriate maximum and minimum points at both ends of the data. Thus, the data sequence must be extended. 

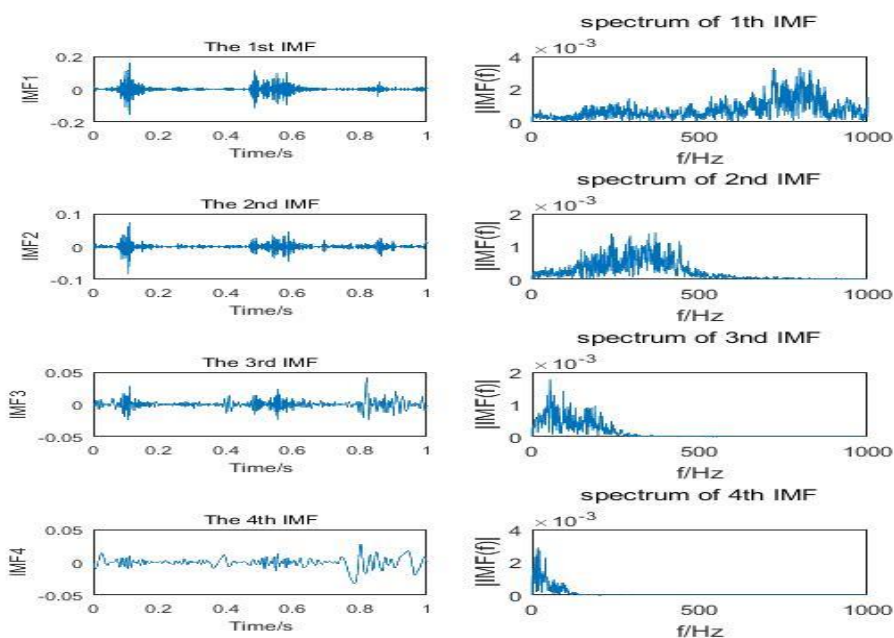

Fig. 3 EMD decomposition results without treatment

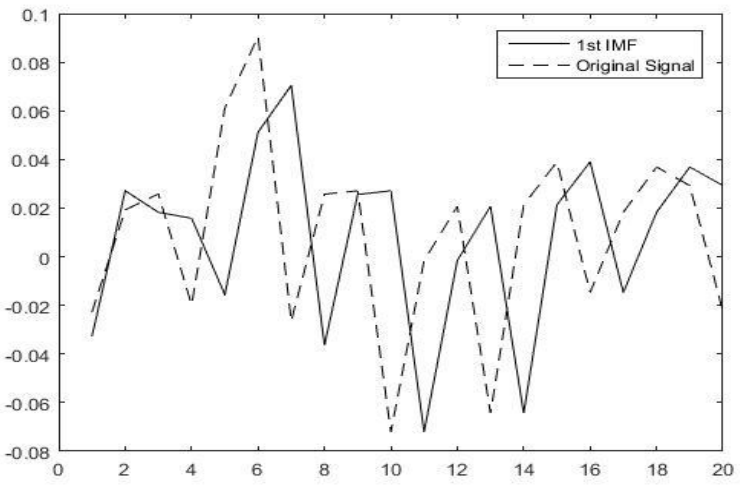

Fig. 4 The first IMF of the signal is compared to the original signal

\subsection{Improvement of EMD Method Based on Even Sequence and Cosine Window Function of Signal Sequences}

Signal sequence is the continuation of the signal to the endpoint symmetry for the center, the data extension method, also known as symmetric extension.If the original signal is $x(t)$, the extension part of the signal is $e(t)$, coordinate origin along the time axis translation to the endpoint, then:

$$
e(t)=x(-t)
$$

Method is to extend the data end of the three cycles of the signal. Compared with non-extended EMD decomposition and Hilbert spectrum, the effect of decomposition is obviously improved, not only the effect of the two principal components above is reduced, but also the amplitude of false component Value has also been reduced. However, compared with EMD decomposition without end effect, EMD decomposed signal has obvious end effect and false component. However, although the extension of the actual signal is relatively close, but there are still errors. On the extended data plus cosine window function processing, control endpoint effect inward "pollution", get more accurate IMF.

Define Cosine Window Functions:

$$
w(t)=\left\{\begin{array}{lc}
\sin \left[\frac{\pi}{2} \times \frac{\mathrm{t}}{\mathrm{A}}\right] & 0 \leq \mathrm{t} \leq \mathrm{A} \\
1 & \mathrm{~A}<\mathrm{t}<\mathrm{L}-\mathrm{A} \\
\cos \left[\frac{\pi}{2} \times\left(\frac{\mathrm{t}-\mathrm{L}+\mathrm{A}}{\mathrm{A}}\right)\right] & \mathrm{L}-\mathrm{A} \leq \mathrm{t} \leq \mathrm{L}
\end{array}\right.
$$

Where, $\mathrm{L}$ is the length of the signal after the extension, $\mathrm{A}$ is the signal extension at both ends of the extension of the length of the longer. 
The cosine function window "controls" the extension error so that it can not (or at a slower speed) develop into the data internally to ensure proper decomposition of the data in the middle of the signal. First, the signal $y(t)=\langle x(t), \omega(t)\rangle$ is obtained by performing an inner product operation of the extension signal $x(t)$ and the cosine window function.

$$
y(t)=\left\{\begin{array}{lc}
x(t) \sin \left[\frac{\pi}{2} \times \frac{t}{A}\right] & 0 \leq t \leq A \\
x(t) & A<t<L-A \\
x(t) \cos \left[\frac{\pi}{2}\left(\frac{t-L+A}{A}\right)\right] & L-A \leq t \leq L
\end{array}\right.
$$

Then, EMD decomposition of the processed signal $\mathrm{y}$, and then decomposed at both ends of the IMF removed the corresponding extension of A. Finally, the boundary spectral analysis of the IMF after subtraction of the extension is performed. The method takes into account both the extension error and the signal integrity.

Figure 5 shows the EMD decomposition of the even extension and cosine window functions.

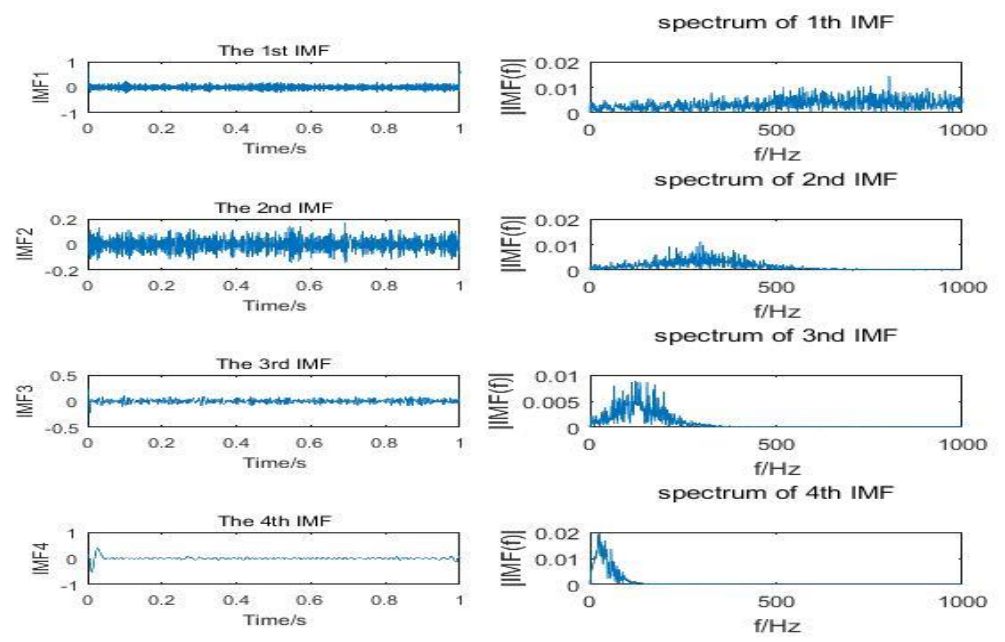

Fig. 5 EMD decomposition results without treatment

Fig. 5 deals with the endpoint effect through even extension and cosine windows compared with Fig. 2, Fig. 6 shows that the IMF obtained by windowing is more consistent with the actual value, which indicates that the method has a good effect on suppressing the endpoint effect.

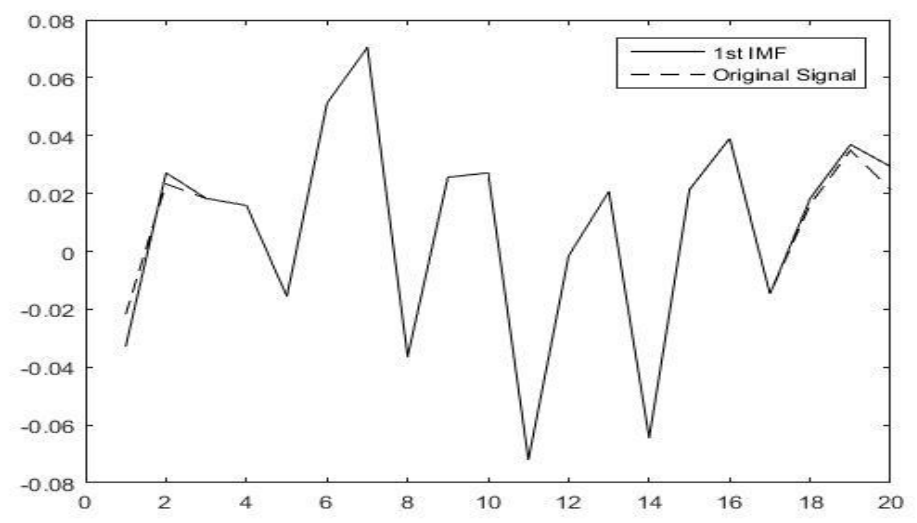

Fig. 6 The first IMF of the signal is compared to the original signal

\section{Support Vector Machines}

In the experiment, five kinds of modes including normal mode were diagnosed and analyzed, with a total of 1000 groups. In the experiment, the failure modes of rotor vibration were analyzed. The corresponding model data distribution is: normal 280; rotor imbalance 230; turn the stator rub: 200; 
shaft system asymmetry: 180; bearing loosening: 110. Wavelet denoising is applied to each of the five data sets, and then the signals are EECW are added. The transformed signals are decomposed by EMD to obtain the eigenvectors of the imf component signals. As a diagnostic sample of the SVM.

In order to more accurately and effectively realize the rotor vibration fault diagnosis, the introduction of SVM can only diagnose the method. First of all, for each model, 50 samples are selected in the training set for training SVM model, then use these data to input SVM model, test and analyze the validity of the model. The correct rate of classification is $100 \%$. And the remaining samples are input to the SVM model as test set. The generalization ability and fault tolerance of this method are tested. The results are shown in Fig1. At the same time, in order to highlight the feasibility and effectiveness of the proposed method, the training samples obtained by EMD decomposition of original signals without any processing are trained in the same way, Testing and diagnosis. The result is compared with the method of SVM diagnosis of even-extended cosine window.

Table 1. Two methods of diagnosis of the rotor

\begin{tabular}{|c|c|c|c|c|c|c|c|c|}
\hline \multirow{2}{*}{$\begin{array}{l}\text { Rotor } \\
\text { Mode }\end{array}$} & \multirow{2}{*}{$\begin{array}{c}\text { Train } \\
\text { Set }\end{array}$} & \multicolumn{2}{|c|}{ Accuracy (\%) } & \multirow{2}{*}{$\begin{array}{l}\text { Test } \\
\text { Set }\end{array}$} & \multicolumn{2}{|c|}{ SVM } & \multicolumn{2}{|c|}{ EECW- SVM } \\
\hline & & SVM & $\begin{array}{c}\text { EECW- } \\
\text { SVM }\end{array}$ & & Correct & $\begin{array}{c}\text { Accuracy } \\
(\%)\end{array}$ & Correct & $\begin{array}{c}\text { Accuracy } \\
(\%)\end{array}$ \\
\hline Normal & 50 & 100 & 100 & 230 & 200 & 86.96 & 216 & 93.91 \\
\hline Unbalanced & 50 & 100 & 100 & 180 & 149 & 82.78 & 166 & 92.22 \\
\hline $\begin{array}{c}\text { Turn Static } \\
\text { Friction }\end{array}$ & 50 & 100 & 100 & 150 & 109 & 72.67 & 140 & 93.33 \\
\hline Misalign & 50 & 100 & 100 & 130 & 98 & 75.38 & 116 & 89.23 \\
\hline $\begin{array}{l}\text { Bearing } \\
\text { Loose }\end{array}$ & 50 & 100 & 100 & 60 & 51 & 85.00 & 55 & 91.67 \\
\hline Average & 50 & 100 & 100 & 750 & 607 & 80.93 & 665 & 92.40 \\
\hline
\end{tabular}

It can be seen from Table 1 that when the training samples are input into the trained SVM model, the correctness of the two methods is $100 \%$, which shows that SVM has better learning ability; from the diagnosis results of the strange samples, the accuracy of the proposed method is $92.40 \%$ based on EECW-SVM. Significantly higher than the SVM direct diagnosis accuracy of 80.93 high. This shows that the SVM directly on the vibration signal of the original data to diagnose the effect of poor, however, the use of wavelet denoising on the original data processing, the use of EECW for endpoint elimination and then emd decomposition Feature can better reflect the original signal characteristics, as a diagnostic sample will make the diagnosis of SVM better.

\section{Conclusion}

A new method of suppressing the endpoint effect is proposed. Firstly, the two ends of the signal are extended by the signal sequence pair extension, then the cosine window is processed according to the extension condition, and the error of the continuation is controlled on both sides. Finally, after EMD decomposition, only the effective length of the signal is taken and the accuracy of decomposition is improved. The signal is denoised by wavelet, the IMF component is obtained by improved EMD decomposition, and the IMF component is used as the feature vector to classify the SVM classifier.Experimental results show that the method can obtain the true and useful signal from the nonlinear fault signal The fault information of rotating machinery can be effectively diagnosed.

\section{References}

[1] Kou H J, Yuan H Q, Zhao X Y. Application of Wavelet De-Noising in Fault Diagnosis of Wind Turbine Gearboxes[C]//Advanced Materials Research. Trans Tech Publications, 2012, 562: 1091- 1094. 
[2] Wu F, Qu L. An improved method for restraining the end effect in empirical mode decomposition and its applications to the fault diagnosis of large rotating machinery[J]. Journal of Sound and Vibration, 2008, 314(3): 586-602.

[3] Yu D, Cheng J, Yang Y. Application of EMD method and Hilbert spectrum to the fault diagnosis of roller bearings[J]. Mechanical systems and signal processing, 2005, 19(2): 259-270.

[4] Cheng J, Yu D, Tang J, et al. Application of SVM and SVD technique based on EMD to the fault diagnosis of the rotating machinery[J]. Shock and Vibration, 2009, 16(1): 89-98.

[5] Feng Z, Liang M, Chu F. Recent advances in time-frequency analysis methods for machinery fault diagnosis: a review with application examples[J]. Mechanical Systems and Signal Processing, 2013, 38(1): 165-205.

[6] Lin D C, Guo Z L, An F P, et al. Elimination of end effects in empirical mode decomposition by mirror image coupled with support vector regression[J]. Mechanical Systems and Signal Processing, 2012, 31: 13-28.

[7] Widodo A, Yang B S. Support vector machine in machine condition monitoring and fault diagnosis [J]. Mechanical systems and signal processing, 2007, 21(6): 2560-2574.

[8] Lei Y, Lin J, He Z, et al. A review on empirical mode decomposition in fault diagnosis of rotating machinery[J]. Mechanical Systems and Signal Processing, 2013, 35(1): 108-126.

[9] Widodo A, et al. Fault diagnosis of low speed bearing based on relevance vector machine and support vector machine[J]. Expert Systems with Applications, 2009, 36(3): 7252-7261.

[10] Hu Q,et al. Fault diagnosis of rotating machinery based on improved wavelet package transform and SVMs ensemble [J]. Mechanical Systems and Signal Processing, 2007, 21(2): 688-705. 